\title{
Gonadotrophin-releasing hormone analogues: a novel treatment for premenstrual asthma
}

\author{
R.D. Murray*, J.P. New*, P.V. Barber**, S.M. Shalet*
}

Gonadotrophin-releasing hormone analogues: a novel treatment for premenstrual asthma. R.D. Murray, J.P. New, P.V. Barber, S.M. Shalet. (C)ERS Journals Ltd 1999.

ABSTRACT: Premenstrual exacerbation of asthma, as reflected by a reduction in peak expiratory flow rate (PEFR), has been demonstrated in $40-100 \%$ of female asthmatics. Epidemiological data demonstrate that admission to hospital with an exacerbation of asthma occurs more frequently perimenstrually. Therapeutic interventions aimed at modifying this precipitating factor, however, remain limited.

We report on a 32-yr old female with asthma in whom a marked increase in symptoms and reduction in PEFR occurred premenstrually, necessitating recurrent admissions to hospital. Frequent severe exacerbations resulted in the chronic use of oral maintenance corticosteroids. In order to suppress gonadotrophin secretion and ovarian function, a long-acting gonadotrophin-releasing hormone analogue was administered with a view to inducing a reversible menopause. This resulted in improvement in respiratory symptoms, the absence of PEFR dips premenstrually, a reduction in maintenance prednisolone dosage and no further hospital admissions during a follow-up period of 14 months.

The authors propose that gonadotrophin-releasing hormone-analogue therapy is a rational and innovative adjuvant treatment worthy of further study in cases of severe premenstrual asthma.

Eur Respir J 1999; 14: 966-967.
*Dept of Endocrinology, Christie Hospital, Manchester, and **Dept of Chest Medicine, Wythenshawe Hospital, Manchester.

Correspondence: S.M. Shalet

Dept of Endocrinology

Christie Hospital NHS Trust

Wilmslow Road

Manchester M23 9LT

UK

Fax: 4401614463772

Keywords: Gonadotrophin-releasing hormone agonists

premenstrual asthma

Received: February 251999

Accepted after revision May 151999
Premenstrual exacerbation of asthma, as reflected by a reduction in peak expiratory flow rate (PEFR), has been demonstrated in $40-100 \%$ of asthmatics $[1,2]$. Clinically, this may remain silent if the deterioration in respiratory function is minor or respiratory reserve is great, and therefore may not result in limitation of normal activity. Further supportive evidence for the importance of menstrual cyclicity in airway reactivity is derived from epidemiological data, demonstrating that admission to hospital with exacerbation of asthma occurs more frequently perimenstrually [3].

Therapeutic strategies aimed at modifying this precipitating factor are currently limited; BEYNON et al. [4] described the successful use of intramuscular progesterone, as demonstrated by an improvement in PEFR and reduction in maintenance prednisolone dosage, in three cases of severe premenstrual asthma. For asthma severity to be cyclical, it is likely to be related to the fluctuations in or absolute concentrations of oestrogen or progesterone. It could therefore be hypothesized that gonadotrophin-releasing hormone (Gn$\mathrm{RH}$ ) analogues by suppression of gonadotrophin and ovarian function, with a view to inducing a premature but reversible menopause, would result in elimination of the premenstrual deterioration in asthma control.

\section{Case study}

A 32-yr old female with a long-standing history of asthma presented with a 4-yr history of worsening symptoms requiring continuous treatment with oral prednisolone. Three years prior to presentation she had noted that the exacerbations were temporally related to her menstrual cycle, occurring during the second week of the luteal phase, reflected in increased wheezing, shortness of breath and a reduction in PEFR from a mean of 400 to $150 \mathrm{~L} \cdot \mathrm{min}^{-1}$. Dramatic improvement followed the commencement of menses (fig. 1). Stabilization of her asthma over this period required the use of oral steroids (prednisolone 7.5-50 mg daily), budesonide nebules $2 \mathrm{mg}$ b.i.d., salbutamol, salmeterol and oral theophyllines. Despite optimal conventional treatment, she required six emergency admissions during this 3-yr period, all occurring premenstrually.

Owing to the clear relationship between exacerbations of her asthma and her menstrual cycle, therapy with goserelin, a potent GnRH-agonist analogue, was commenced in order to suppress her reproductive axis. This resulted in amenorrhoea and undetectable gonadotrophin (luteinizing hormone $<3$ IU. $\mathrm{L}^{-1}$, follicle-stimulating hormone $<3 \mathrm{IU} \cdot \mathrm{L}^{-1}$ ) and oestradiol $\left(<100 \mathrm{pmol} \cdot \mathrm{L}^{-1}\right)$ levels. During the initial 6 months of adjuvant therapy with goserelin, a significant improvement was observed in asthma symptoms and PEFR. Minimal maintenance oral steroid therapy was required (prednisolone $5 \mathrm{mg}$ o.d.). Estimation of bone mineral density, using dual x-ray absorptiometry, prior to $\mathrm{GnRH}$ analogue treatment, confirmed the presence of osteopenia believed to be the result of prolonged corticosteroid therapy. Hormone replacement therapy (HRT) in the form of conjugated equine oestrogen was, therefore commenced. HRT caused recurrence of the premenstrual exacerbation of her asthma as evidenced by a reduction in mean PEFR from 400 to $300 \mathrm{~L} \cdot \mathrm{min}^{-1}$, recurrence of symptoms and an increased requirement in maintenance prednisolone dosage (5-30 mg. day ${ }^{-1}$ ), culminating in an emergency admission with asthma. The HRT was withdrawn whilst the goserelin was continued. Her asthma again improved, reflected 


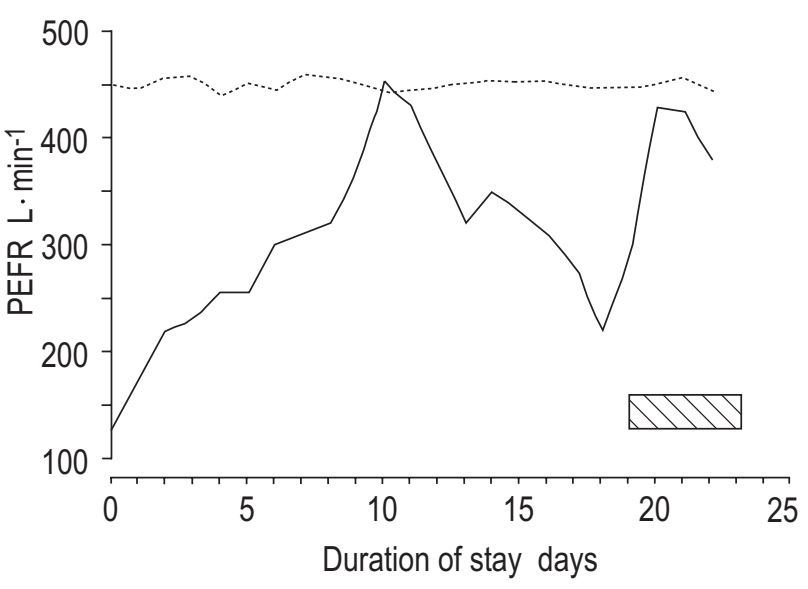

Fig. 1. - Peak expiratory flow rate (PEFR) following an acute admission for asthma demonstrating a typical premenstrual dip ( -$) ; \mathbb{\mathbb { N }}$ : menses and following treatment with a gonadotrophin-releasing hormone analogue $(---)$; whilst amenorrhoeic.

by a reduction in symptoms, maintenance prednisolone dose and number of days off work and an increase in mean PEFR to $450 \mathrm{~L} \cdot \mathrm{min}^{-1}$. At the last review, she remained well, having completed a further 8 months on goserelin. Significantly, during the 14 months of treatment with goserelin alone there were no hospital admissions.

\section{Discussion}

In this female, there is a marked relationship between asthma and menstrual cycle. To the authors' knowledge this is the first case of uncomplicated premenstrual asthma being successfully treated with a GnRH analogue. GIBBS et al. [1] demonstrated that $40 \%$ of females experience premenstrual symptomatic exacerbation of their asthma, confirmed by PEFR findings. A more recent study in 17 female asthmatics showed premenstrual worsening of symptoms with a reduction in PEFR of $>20 \%$ in all those studied, although only $36 \%$ had noted any effect of their menstrual cycle on their asthma at enrolment [2]. SkOBeloff et al. [3] reported an increased frequency of exacerbations of asthma necessitating hospital treatment during the perimenstrual phase of the cycle.

The mechanism of premenstrual deterioration is poorly understood; for asthma seventy to be cyclical, it is likely to be related to the fluctuations in or absolute concentrations of oestrogen or progesterone. It has been suggested that higher oestradiol levels during a cycle are associated with improvement in asthma symptoms and that the clinical deterioration reflects an abrupt fall in oestradiol levels [2]. In addition, progesterone is recognized to have a relaxant effect on smooth muscle and may thus contribute to the cyclical changes in airway responsiveness seen in menstrual-related asthma [5]. By administration of a GnRH analogue, gonadotrophin release is attenuated; the loss of gonadotrophin drive results in failure of the ovary to secrete oestrogen or progesterone. The induced absence of cyclical fluctuations in oestrogen and progesterone results in amenorrhoea and absence of the cyclical deterioration in asthma control. BEYNON et al. [4] described the administration of intramuscular progesterones, either $100 \mathrm{mg}$ daily or $600 \mathrm{mg}$ twice weekly, to three patients with severe premenstrual asthma [4]. This intervention eliminated the premenstrual dips in PEFR and all three patients were able to reduce their prednisolone dosage. Progesterones given in the doses used by BEYNON et al. [4] are not physiological but supraphysiological and thus have a pharmacological effect. High-dose progesterone inhibits gonadotrophin release through negative feedback at the hypothalamopituitary level with consequent oligo- or amenorrhoea. Progesterone therapy thus improves premenstrual asthma by a common final pathway with that of GnRH analogues. GnRH analogues have several distinct advantages over progesterones. Goserelin is administered as a once monthly s.c. injection in contrast to the daily or twice weekly i.m. progesterone injections. Intramuscular injections are frequently painful. On discontinuation of progesterone (DepoProvera), prolonged periods of amenorrhoea may occur. Progesterone therapy is therefore not readily reversible if a patient requires fertility, remembering that pre-menstrual asthma occurs, by definition, in the reproductive years.

Bone loss is a potential problem using both GnRH-analogues and progesterones. Both modes of treatment aim to reduce corticosteroid dependence and thus bone loss caused by this insult. The purpose of add-back HRT whilst on GnRHanalogue therapy is to replace an adequate amount of oestrogen to maintain the skeleton. Clearly, this is worthwhile if the small doses of oestrogen and progestogen constituting the HRT do not influence the asthma. Unfortunately, even small doses of administered cyclical hormones resulted in exacerbation of the patient's premenstrual asthma. The osteopenia of oestrogen deficiency can be treated with bisphosphonates [6]. An alternative strategy would be the use of continuous HRT preparations in order to avoid hormonal fluctuations but this has yet to be explored. Asthma symptoms and hospital attendance greatly reduce the quality of life of the patients and high maintenance doses of steroids result in iatrogenic Cushing's syndrome and osteopenia.

The use of gonadotrophin-releasing hormone analogues may thus be an important adjuvant treatment in selected patients with severe asthma experiencing premenstrual exacerbations. Gonadotrophin-releasing hormone analogues provide a novel approach to a common and not infrequently disabling medical condition and thus warrant further study.

\section{References}

1. Gibbs CJ, Lock R, Finnegan OC, White RJ. Premenstrual exacerbation of asthma. Thorax 1984; 39: 833-836.

2. Chandler MH, Schuldheisz S, Phillips BA, Muse KN. Premenstrual asthma: the effect of estrogen on symptoms, pulmonary function, and beta 2-receptors. Pharmacotherapy 1997; 17: 224-234.

3. Skobeloff EM, Spivey WH, Silverman R, Eskin BA, Harchelroad F, Alessi TV. The effect of the menstrual cycle on asthma presentations in the emergency department. Arch Intern Med 1996; 156: 1837-1840.

4. Beynon HL, Garbett ND, Barnes PJ. Severe premenstrual exacerbations of asthma: effect of intramuscular progesterone. Lancet 1988; 2: 370-372.

5. Boggess KA, Williamson HO, Homm RJ. Influence of the menstrual cycle on systemic diseases. Obstet Gynecol Clin North Am 1990; 17: 321-342.

6. Adachi JD, Bensen WG, Brown J, et al. Intermittent etidronate therapy to prevent corticosteroid-induced osteoporosis. N Engl J Med 1997; 337: 382-387. 